Revue d'histoire de l'Amérique française

REVUE D.HISTOIRE DE L'AMÉRIQUE FRANÇAISE

\title{
Le destin de l’Église sous le régime militaire
}

\section{Marcel Trudel}

Volume 11, numéro 1, juin 1957

URI : https://id.erudit.org/iderudit/301802ar

DOI : https://doi.org/10.7202/301802ar

Aller au sommaire du numéro

Éditeur(s)

Institut d'histoire de l'Amérique française

ISSN

0035-2357 (imprimé)

1492-1383 (numérique)

Découvrir la revue

Citer cet article

Trudel, M. (1957). Le destin de l'Église sous le régime militaire. Revue d'histoire de l'Amérique française, 11(1), 10-41. https://doi.org/10.7202/301802ar d'utilisation que vous pouvez consulter en ligne.

https://apropos.erudit.org/fr/usagers/politique-dutilisation/ 


\section{LE DESTIN DE L'ÉGLISE SOUS LE RÉGIME MILITAIRE *}

Veut-on savoir si la vie de l'Église canadienne sous le Régime militaire a été heureuse ou malheureuse ? L'histoire présente des témoins qui, à première vue, paraissent se contredire. Les uns sont tout éloge; c'est le cas de Montgolfier qui écrit en octobre 1761: «Dans le spirituel tout comme dans le civil, nous ne nous apercevons pas quasi de la révolution »: et Mère d'Youville déclare en août 1763: «Si nous sommes aussi libres d'exercer notre religion et de faire tout le bien que nous trouvons à faire comme nous l'avons été depuis que nous sommes sous la domination anglaise, nous ne serons pas à plaindre pour le spirituel. » D'autres témoins, par contre, s'expriment avec pessimisme; racontant les difficultés qu'il éprouve, le grand vicaire Perrault écrit en mars 1762: "Nous aurons de grands arrengemens a Prendre, bien des Croix et des humiliations a Essuyer, Supposé que le pais Reste a l'angleterre, pour y Soutenir Et deffendre la Religion »; faisant allusion à certains règlements rigoureux imposés par l'Angleterre à l'Église canadienne, un prêtre du Séminaire de Québec écrit: «Et ce qui nous afflige le plus c'est que tous les Règlements sont précisement ceux qu'avoit proposé a la Cour M. de Murray maintenant gouverneur Général du Canada».

Ces appréciations que nous tirons de lettres personnelles et privées (car les documents officiels et publics n'ont point le même son de sincérité), ne sont pas contradictoires; chacune s'applique exactement et uniquement à la région géographique dans laquelle elle a été rédigée ou à une étape déterminée du Régime militaire. Car, et il ne faut pas le perdre de vue, le Canada s'est trouvé divisé en trois Gouvernements ou pays bien distincts où les problèmes politico-religieux n'ont pas été traités d'une façon iden-

* Conclusion générale de L'Eglise canadienne sous le Régime militaire, 1759-1764, conclusion extraite du volume II qui paraîtra prochainement. 
tique; de plus, ces cinq années du Régime militaire ont posé à l'Église des problèmes si complexes qu'une appréciation d'ensemble risque toujours de ne pas être parfaitement valable. Essayons quand même de prendre une vue d'ensemble de la situation.

\section{Les pertes matérielles}

Cette période commence, pour l'Église canadienne, par des dommages matériels et, en certains cas, par une destruction d'églises et de presbytères. Certes, les chefs de l'armée anglaise ont donné des ordres précis pour que les églises soient respectées, mais comment les 140 églises ou chapelles pouvaient-elles toutes échapper aux hasards d'une invasion générale ? Comme c'est dans la région de Québec que les opérations militaires se sont prolongées, c'est là surtout que nous rencontrons des églises endommagées ou détruites. Dans la capitale même, après le long siège que l'on sait, la cathédrale, la chapelle du palais épiscopal, la chapelle Notre-Dame-des-Victoires ne sont plus réduites qu'à leurs murs; au Séminaire, chez les Récollets, chez les Ursulines et chez les Jésuites, les chapelles subissent des dommages assez élevés. Dans la campagne de Québec, les cas de destruction totale sont rares: l'église de Saint-Joachim est détruite par représailles contre les habitants qui pratiquent la petite guerre; celle de Sainte-Foy est dynamitée pour des raisons stratégiques, comme tout probablement celle de l'Ancienne-Lorette; ailleurs, les dommages sont plus ou moins élevés selon que les églises se trouvaient situées plus ou moins près du champ des opérations militaires. Sept des 64 églises ou chapelles du Gouvernement de Québec demeurent hors d'usage; une quinzaine d'autres sont endommagées. Dans le Gouvernement des Trois-Rivières, une seule des 20 églises est détruite, et ce par un parti de guerre qui opère isolément en 1759. Des 48 temples du Gouvernement de Montréal, seuls ceux de Sorel, de Varennes, de Chambly et de Châteauguay subissent quelque dégât. Dans la région des Grands Lacs et des Illinois, où nous trouvons 8 chapelles, il n'y a rien à noter. En somme, de ces 140 églises ou chapelles,

$\begin{array}{rlll}8 & \text { soit } & 5.7 \% & \text { restent hors d'usage } \\ 18 & \text { soit } 12.9 \% & \text { sont lourdement endommagées } \\ 7 & \text { soit } 5 \% & \text { ont subi des dommages mineurs } \\ 107 & \text { soit } 76.4 \% & \text { sortent intactes de la guerre. }\end{array}$


Si nous évaluons les pertes matérielles subies par les presbytères, c'est encore dans la région de Québec que nous en trouvons le plus: sur environ 51 presbytères, il en disparaît au moins 14 ; dans la région des Trois-Rivières, il n'en disparaît qu'un seul; de même, dans la région de Montréal. Des 120 presbytères que nous connaissons, 16 sont incendiés, soit une proportion de $13.13 \%$, et un nombre d'autres indéterminé ont été plus ou moins endommagés: les autorités militaires ne s'étaient pas préoccupées d'assurer aux presbytères la même protection qu'aux églises. Vues dans l'ensemble du pays, ces pertes matérielles en églises et presbytères ne présentent qu'une faible proportion, $25 \%$ au maximum. Remarquons bien, cependant, que la plus grande partie de ces pertes se produisent dans une même région, le Gouvernement de Québec, et que les habitants y ont, en même temps, à relever leurs habitations: pour la population de cette région, le poids de la guerre est vraiment écrasant.

Cette Église éprouve encore des difficultés matérielles dans ses Fabriques paroissiales. Nous avons pu étudier la situation financière de 27 de ces Fabriques: or, en plus du désordre que la guerre cause dans certaines archives, nous remarquons que les recettes et dépenses diminuent à mesure que s'étend l'occupation anglaise, diminution due au contrôle des prix qui reviennent à la normale et surtout à l'argent de papier que l'on retire peu à peu de la circulation; pour sa part, le produit des quêtes subit une chute soudaine au moment de la soumission générale du pays, cette chute s'accélère en 1762 , puis la courbe reprend sa montée en 1763. Ce sont, le plus souvent, des dépenses extraordinaires qui absorbent la portion importante des revenus: réparation des dommages dûs à la guerre, construction ou réfection de l'église, dépenses spéciales pour le presbytère, cotisation pour l'ambassade de Charest en Angleterre. Il arrive que certaines Fabriques florissantes se procurent, même sous le régime militaire, des articles de luxe qu'elles paient argent comptant, mais ce sont là des cas vraiment exceptionnels. Même si, sur 27 Fabriques que nous avons spécialement étudiées, 5 seulement déclarent des déficits à peu près chaque année, les difficultés matérielles restent générales à presque toutes: on dépense fort 
peu parce que les recettes sont fort maigres. La crise du papier sème le désarroi dans les Fabriques comme ailleurs: à une époque où ce papier n'a plus cours, les fidèles continuent d'en donner à la quête. Les Fabriques trouvent parfois le moyen de s'en servir à l'achat de divers articles, mais cela ne diminue guère l'amoncellement de l'argent mort. C'est la Fabrique de Notre-Dame-deMontréal qui en détient le plus : 21,002 livres 10 sols; elle pouvait se compter chanceuse si, dans cette débâcle, elle sauvait 5,000 livres! La majorité des Fabriques ont cependant moins de 4,000 livres d'argent de papier; quant au grand total, environ 220,000 livres, il peut paraître tout à fait négligeable dans les quelque 16,800,000 livres de papier que l'on trouve au Canada: les Fabriques ne détiennent que $1.3 \%$ de tout l'argent de papier. Toutefois, il faudrait en ce cas les considérer une à une: pour une Fabrique qui dépense peu parce que ses recettes sont pauvres, la perte de 1,000 ou 2,000 livres est considérable.

\section{Trois Communautés de femmes en péril}

De cet aspect strictement matériel, si nous passons aux ressources humaines de l'Église canadienne, nous nous rendons bien compte qu'en raison de circonstances complexes cette période du Régime militaire porte un dur coup aux institutions religieuses.

En 1759, on trouve sept Communautés de femmes au Canada: à Québec, les Ursulines, les Hospitalières de l'Hôtel-Dieu, les Hospitalières de l'Hôpital Général, les Sœurs de la Congrégation de Notre-Dame; aux Trois-Rivières, les Ursulines; à Montréal, les Hospitalières de l'Hôtel-Dieu, les Sœurs Grises de l'Hôpital Général, les Sœurs de la Congrégation de NotreDame. De plus, ces mêmes Sœurs de la Congrégation tiennent école en divers autres endroits: Lac-des-Deux-Montagnes, Lachine, Saint-Laurent, la Pointe-aux-Trembles (près Montréal), Laprairie, Boucherville, Champlain, la Pointe-aux-Trembles (Neuville), Château-Richer, Sainte-Famille en l'île d'Orléans.

Les opérations militaires dérangent bien des religieuses. A Québec, les Sœurs de la Congrégation doivent quitter leur couvent de la basse-ville; les Ursulines et les Hospitalières de l'Hôtel- 
Dieu vont se réfugier à l'Hôpital Général qui devient vite surpeuplé de civils, de militaires français et anglais. Le siège terminé, les religieuses retournent chez elles. Les Ursulines trouvent leur monastère endommagé, moins cependant que d'autres édifices de premier plan; Murray le fait réparer à la hâte pour que ses blessés trouvent un abri et pour que les protestants de concert avec les paroissiens de Notre-Dame, puissent utiliser la chapelle; et ainsi, pendant des mois, les Ursulines servent d'hospitalières et prêtent leur temple au culte protestant; quant aux paroissiens de Notre-Dame, ils viennent à cette chapelle, église paroissiale temporaire, jusqu'en décembre 1764. L'Hôtel-Dieu a été endommagé lui aussi, mais la maison reste logeable; la vie reprend donc, mais avec un changement notable: l'Hôtel-Dieu sert désormais d'hôpital militaire à l'usage exclusif des Anglais. Quant au couvent de la basse-ville, tenu par les Sœurs de la Congrégation, il n'est plus que ruines. Un autre couvent de la Congrégation disparaît dans la tourmente: celui du Château-Richer, que les Anglais détruisent après l'avoir occupé; celui de Sainte-Famille est sauvé de justesse, mais les religieuses ont été obligées de fuir; à la Pointe-aux-Trembles (Neuville), les Anglais font une descente et capturent une religieuse avec huit pensionnaires, on les libère dès le lendemain; le couvent sert de casernes aux Français d'abord, puis aux Anglais jusqu'en 1761. Ailleurs, où l'armée anglaise ne fait en somme que recueillir la soumission des habitants, il ne se produit aucun dérangement important. A Montréal, les religieuses n'éprouvent aucune perte matérielle; rien ne change, sauf pour l'Hôtel-Dieu qui reçoit des soldats anglais et dont la chapelle servira au culte protestant jusque vers 1768 .

A l'automne de 1760 , nous trouvons dans la vallée du SaintLaurent (sans tenir compte des sœurs converses) un total de 204 religieuses ainsi réparties:

Congrégation de Notre-Dame

Ursulines de Québec

Hôtel-Dieu de Québec

Hôtel-Dieu de Montréal

Hôpital Général de Québec

$\begin{array}{cc}\text { nombre } & \text { âge moyen } \\ 70 & 48.7 \\ 30 & 45 . \\ 26 & 45.6 \\ 26 & 35.7 \\ 22 & 35.1 \\ 15 & 40.7 \\ 15 & 40.5\end{array}$

Ursulines des Trois-Rivières

15

40.5 
La plus nombreuse Communauté de femmes est celle de la Congrégation, qui compte 70 religieuses: elles ne sont pas cloîtrées, elles ont un régime moins sévère et on n'exige des candidates qu'une dot de 2,000 livres; ce qui explique leur population élevée. Quant aux Sœurs Grises qui, elles non plus, ne sont pas cloîtrées, leur petit nombre vient de ce que la fondation de la Communauté est toute récente. La Congrégation de Notre-Dame, qui est la Communauté la plus nombreuse, est aussi celle qui a la moyenne d'âge la plus élevée: 48.7 ans, mais par contre elle est dirigée par la plus jeune Supérieure de tout le pays: cette Supérieure n'a que 50 ans. Chez ces 204 religieuses, nous ne rencontrons pas une seule Française de France: les Communautés de femmes sont devenues tout à fait canadiennes.

Si l'on s'arrête à leur origine sociale, c'est l'Hôpital Général de Québec qui prend figure de couvent «chic» de la NouvelleFrance:

Hôpital Général de Québec

Hôtel-Dieu de Montréal

Ursulines de Québec

Hôpital Général de Montréal

Ursulines des Trois-Rivières

Hôtel-Dieu de Québec

Congrégation de Notre-Dame nombre total des religieuses

22

26

30

15

15

26

70 pourcentage des filles de grandes familles

$59.1 \%$

$30.6 \%$

20

20

$13.3 \%$

$11.6 \%$

$10 \%$

Si l'on ne retient que les filles de chevaliers de Saint-Louis, la comparaison est encore plus frappante: alors que $40.9 \%$ des religieuses de l'Hôpital Général de Québec sont filles de chevaliers de Saint-Louis, ce même pourcentage dans les deux Communautés qui viennent immédiatement au second rang (Ursulines de Québec et Ursulines des Trois-Rivières) n'est plus que de 6.7; il est nul à l'Hôtel-Dieu de Québec et à l'Hôpital Général de Montréal. La Communauté la plus aristocratique (sans toutefois donner à ce mot toute son acception française) est donc celle de l'Hôpital Général de Québec; celle qui fait le plus «peuple»est la Congrégation de Notre-Dame.

La capitulation de Montréal s'est montrée généreuse pour les Communautés de femmes: non seulement elle leur garantit comme à tout le monde la possession des biens, mais elle accepte 
même de les conserver dans leurs constitutions et privilèges. D'autres gestes des autorités d'occupation contribuent à faire sentir moins durement les dégâts de la guerre.

En effet, chez les Ursulines de Québec, le gouverneur Murray ordonne, aux frais du Roi, les réparations essentielles. Murray a besoin de locaux pour ses blessés et d'une chapelle pour les protestants, mais, en retour, les Ursulines reçoivent gratuitement des rations de l'armée, depuis l'automne de 1759 jusqu'au printemps de 1761; leurs services d'hospitalières sont rémunérés: de novembre 1759 à juillet 1760 , elles touchent en argent sonnant 4,314 livres. Par la suite, les relations avec les Anglais demeurent toujours cordiales, relations sans doute facilitées par l'élection en décembre 1760 d'une Supérieure née dans les colonies anglaises; et quand les Ursulines rouvrent leurs classes, des protestants (même le ministre Brooke) y envoient leurs fillettes.

L'Hôtel-Dieu de Québec jouit aussi de la protection des Anglais. En se réservant l'usage exclusif de cet hôpital, les Anglais assurent la subsistance des religieuses: de 1759 à 1764, elles touchent pour elles-mêmes ou pour les Pauvres 12,779 livres 12 sols 6 deniers; le Commissariat des Vivres nourrit gratuitement la Communauté, du 21 septembre 1759 à janvier 1760 ; les Anglais envoient ensuite des vivres pour une valeur d'au moins 2,600 livres, outre 844 livres en aumônes. Enfin, le gouverneur Murray autorise les religieuses à garder les 3,389 livres 4 deniers qu'elles avaient perçues par erreur à la place du Domaine du Roi. A ce compte, l'occupation de la maison par l'armée s'avérait profitable.

L'Hôpital Général de Québec reçut moins de faveurs, parce que ses services avaient été moins prolongés: les Anglais quittent cette maison dès l'été de 1760 , mais ils laissent tout de même 6,500 livres pour acquitter les frais d'hospitalisation. A l'adresse des autres Communautés, les faveurs en argent sonnant sont rares. Les Ursulines des Trois-Rivières n'ont peut-être rien reçu. A Montréal, Amherst visite la Congrégation de Notre-Dame, sans laisser, semble-t-il, de souvenirs tangibles; d'ailleurs, les Sœurs n'ont ni logé ni soigné de soldats anglais. L'Hôpital Général des Sœurs Grises loge des soldats, mais nous ignorons s'ils sont 
Anglais ou Français (les comptes manquant de précisions) ; pour l'œuvre des enfants trouvés, le gouverneur Gage donne 288 livres qui viennent peut-être d'amendes perçues; les cadeaux se laissent désirer et Mère d'Youville se plaint que les Anglais n'accordent point de contrats d'ouvrages comme le faisaient les Français. L'Hôtel-Dieu de Montréal, qui loge des soldats anglais, est quelque peu favorisé: lors de sa visite, Amherst laisse un montant de 1,200 livres françaises et ... deux douzaines de bouteilles de Madère; en 1763, Gage envoie une barrique de vin; là aussi, les Anglais paient les frais d'hospitalisation: de 1762 à 1764, ils donnent au moins 1,460 livres 12 sols (nous disons au moins, parce que nous avons là un estimé incomplet). Nous ignorons ce qu'ils ont donné pour l'usage de la chapelle.

$\mathrm{Au}$ point de vue financier, ce sont les Ursulines de Québec qui semblent se tirer d'affaires le moins péniblement: pour cette période de cinq ans, le déficit est seulement de 6,439 livres 2 sols 6 deniers et, lors de la liquidation du papier, elles ne perdent que 14,250 livres: perte et déficit qui apparaissent plutôt négligeables quand on considère ce qui se passe dans d'autres Communautés. Nous connaissons mal la situation financière des Ursulines des Trois-Rivières, faute de documents; nous savons seulement qu'elles perdent d'un coup, en argent de papier, près de 30,000 livres et, comme elles avaient été obligées de se reconstruire en 1752 , nous pouvons déduire qu'elles sont en ce moment accablées de lourdes dettes. Mal connue aussi la situation financière de la Congrégation de Notre-Dame, toujours faute de documents; nous savons, cependant, que la Communauté perd, dans la crise de l'argent de papier, environ 40,000 livres. L'Hôpital Général des Sœurs Grises perd à cause du papier une somme d'environ 80,000 livres. N'eût été cette liquidation malheureuse, l'Hôpital se fût tiré d'affaires fort avantageusement: il termine, en effet, le régime militaire par un surplus de 37,000 livres, cependant que Mère d'Youville trouvait le moyen d'augmenter les biens-fonds de son institution; la perte de 80,000 livres restait quand même en l'occurrence un dur coup.

Trois Communautés touchent de très près la catastrophe. L'Hôtel-Dieu de Montréal avait perçu jusqu'en septembre 1760 
un revenu mensuel de 4,000 livres; or, pendant les années 17621764, ce revenu mensuel n'est plus que de 80 livres! Les dettes de la maison ne sont pas élevées (7,000 livres environ), mais l'hôpital perd 6,000 livres en argent de papier et les religieuses y perdent, pour leur part, 79,250 livres. On comprend ainsi que, dans un moment de désespoir, les hospitalières aient songé à fermer la maison et à se réfugier en France. Même situation accablante à l'Hôtel-Dieu de Québec: au moment de l'invasion, cette maison est endettée de 106,614 livres, par suite de la reconstruction; chaque année du régime militaire reste déficitaire, ce qui amène un déficit global de 8,399 livres; à la fin de 1764, l'Hôtel-Dieu doit encore près de 100,000 livres, il ne peut plus compter sur les 7,500 livres qu'il recevait du Roi en gratifications annuelles et la liquidation de l'argent de papier lui fait perdre 26,000 livres ! Mais c'est l'Hôpital Général de Québec qui, de toutes les Communautés de femmes, subit le plus durement les événements de la conquête. Avant l'invasion, la Communauté n'avait qu'une dette négligeable $(9,358$ livres $)$ et la maison elle-même n'a pas souffert de la guerre; le désastre vient d'ailleurs. C'est que, d'une part, pour secourir les blessés français, la Communauté avait été obligée de s'endetter: en 1761, cette dette se chiffre à 50,000 livres; d'autre part, l'Hôpital Général possède un amoncellement d'argent de papier: 131,846 livres. Pour répondre aux créanciers les plus pressants, il fallut emprunter (ce n'était pas résoudre le problème financier); puis on connut les conditions de la liquidation: la Communauté perdait d'un coup environ 100,000 livres. La Supérieure écrira: "Nous sommes la seule communauté religieuse de ce pays exposée à mendier son pain ». Là aussi, on songea sérieusement à fermer la maison.

Pendant cette période du régime militaire, le recrutement des religieuses est fort maigre: pour les sept Communautés de femmes, nous ne trouvons en cinq ans qu'un total de 22 recrues. La situation est tout à fait alarmante dans deux Communautés (d'ailleurs menacées de la faillite) : à l'Hôtel-Dieu de Québec, il ne se présente aucune nouvelle Sœur converse pendant une période de 10 ans; à l'Hôpital Général de Québec, on n'en voit pas arriver une seule pendant 21 ans et la Communauté ne reçoit, 
en 9 ans, qu'une seule religieuse de chœur. Baisse considérable du recrutement et, de plus, nombre relativement élevé des décès : il meurt au pays, en l'espace de cinq ans, un total de 42 religieuses de chœur. A l'automne de 1760, nous avions compté 204 religieuses; il en reste 190 à l'automne de 1764 :

Congrégation de Notre-Dame

Ursulines de Québec

\begin{tabular}{cccc}
\multicolumn{2}{c}{ nombre } & \multicolumn{2}{c}{ âge moyen } \\
1760 & 1764 & 1760 & 1764 \\
70 & 61 & 48.7 & 48.4 \\
30 & 31 & 45 & 46.6 \\
26 & 25 & 45.6 & 44.1 \\
26 & 22 & 35.7 & 36.8 \\
22 & 21 & 35.1 & 37.1 \\
15 & 16 & 40.7 & 41.1 \\
15 & 14 & 40.5 & 39.5
\end{tabular}

Hôtel-Dieu de Québec

Hôtel-Dieu de Montréal

Hôpital Général de Québec

Hôpital Général de Montréal

Ursulines des Trois-Rivières

La Congrégation de Notre-Dame demeure la plus nombreuse Communauté de femmes, mais la marge s'est quelque peu rétrécie entre ces religieuses séculières et les Ursulines cloîtrées. C'est encore la Congrégation qui a la moyenne d'âge la plus élevée. En 1760, la Communauté la plus avancée en âge était dirigée par la plus jeune Supérieure du pays; en 1764, la situation est renversée: les deux plus jeunes Communautés (l'Hôtel-Dieu de Montréal et l'Hôpital Général de Québec) ont à leur tête les plus vieilles Supérieures... Le niveau social s'est considérablement modifié là où en 1760 il était élevé: à l'Hôpital Général de Québec, la proportion des filles de grandes familles tombe de $59.1 \%$ à $52.4 \%$; à l'Hôtel-Dieu de Montréal, elle tombe de $30.6 \%$ à $18.2 \%$ ! L'Hôtel-Dieu de Montréal retourne rapidement à la roture; quant à l'Hôpital Général de Québec, couvent «aristocratique » du pays, il modifie déjà son visage. Pour les sept Communautés de femmes, le pourcentage "aristocratique » était en 1760 de 20.6; il est tombé à 17.9 en 1764 : l'ensemble des religieuses du pays va bientôt devenir «peuple».

\section{Chanoines, Jésuites et Récollets quittent la scène}

Les Communautés de femmes ont donc subi, pendant cette période, une dure décadence: les effectifs ont diminué et, en certains cas, la situation financière est devenue quasi catastrophique. Chez les Communautés d'hommes (composées de prêtres, donc 
de membres essentiels à la survivance de l'Église), nous trouvons un état de choses bien plus grave encore: le Séminaire de Québec, écrasé par la guerre, devra prendre une orientation nouvelle; et trois Communautés (le Chapitre, les Jésuites, les Récollets) seront appelés à disparaître par suite des circonstances de la conquête.

Déjà, au moment de l'invasion (car la conquête n'est pas responsable de tout), le Chapitre subissait la décadence: sur 12 chanoines, il n'y en avait plus que 9 au pays; et encore sur ces 9 chanoines, il n'en restait plus que 6 à Québec pour réciter l'office canonial, fonction primordiale et presque exclusive du Chapitre. Le bombardement ayant détruit la cathédrale, le Chapitre n'a plus ni toit ni chœur; deux chanoines canadiens s'en vont en France; il en meurt un autre, ce qui réduit les chanoines à 6 , dont seulement 3 à Québec. Ce Chapitre, héritier du siège épiscopal, ne désigne point de vicaire capitulaire et laisse aux grands vicaires le soin d'administrer l'Église; il demeure dispersé sans reprendre en chœur la récitation de l'office. Il joue tout de même un rôle de premier plan lorsque, dans une tentative pour régler le problème épiscopal, il s'arroge en septembre 1763 le droit d'élire l'évêque: celui qu'ils choisissent, Montgolfier, est agréé en Angleterre; Rome, sans reconnaître l'élection du Chapitre, est prête aussi à accepter Montgolfier; or, à cause de l'opposition de Murray, le Chapitre doit recommencer l'élection en septembre 1764 et désigne Briand à l'épiscopat. Jamais les chanoines du Canada n'avaient joué un rôle aussi important, mais un autre problème très grave se posait à ces mêmes chanoines: celui de leur conservation. On avait proposé que le Chapitre fût à l'avenir chargé d'élire l'évêque et Mgr Briand, pour sa part, proposa que ce Chapitre fût au moins chargé de choisir le coadjuteur: Rome refusa tout à fait aux chanoines le droit d'élire l'évêque; quant au coadjuteur, c'est à Mgr Briand lui-même qu'en fut laissé le choix. Dans ces conditions, le Chapitre cessait d'être (ce qu'il n'était que depuis 1760) une institution essentielle. Et puis, comment pouvait-il désormais subsister ? Les chanoines ne touchent plus les 8,000 livres annuelles du Domaine du Roi, on leur enlève l'abbaye française de Maubec; 
leur seigneurie de la Petite-Nation ne rapporte pas un sol. De plus, l'institution canoniale est en voie rapide d'extinction: à la fin de 1764, sur les 10 chanoines qui composent le Chapitre, il n'en reste plus que 5 au Canada, vivant toujours dans la dispersion, sans jamais reprendre en chœur la récitation de l'office; on ne remplit pas les vacances: c'est à peine si les chanoines se réunissent de temps à autre pour discuter des ressources matérielles. Il n'y a plus de Chapitre, il n'y a que des chanoines; leur histoire n'intéresse plus l'Église. Les contemplatifs, établis par Mgr de Laval, disparaissent comme Communauté, victimes à la fois de leurs propres misères et des événements de la conquête.

Une deuxième Communauté d'hommes va disparaître: celle des Jésuites. Dès les débuts du régime militaire, la vie devient difficile pour eux: le collège qu'ils dirigent à Québec est réquisitionné pour servir d'entrepôt à l'armée; mesure d'urgence qui reste légitime à condition d'être temporaire, mais cette mesure deviendra permanente. Quelques jésuites qui se compromettent personnellement pendant les opérations militaires, contribuent peut-être à augmenter les soupçons des vainqueurs protestants. En tout cas, la capitulation générale refuse de se prononcer sur la conservation des Jésuites, même si elle leur reconnaît la propriété des biens. Et les Jésuites diminuent rapidement en nombre: en 1759 et en 1760 , ils perdent 6 prêtres, 3 scolastiques et 2 Frères; à la fin de 1760 , ils ne sont plus que 25 prêtres, âgés en moyenne de 50.4 ans, ce qui en fait la Communauté la plus avancée en âge; de plus, ils viennent tous d'Europe, ce qui n'est pas de nature à les aider. A mesure que les événements se déroulent, les Jésuites deviennent de moins en moins sûrs de l'avenir. En effet, ils perdent à Montréal en 1762 un important procès: les autorités militaires décident qu'ils ne sont pas les seigneurs du Sault-SaintLouis. En 1763, le gouverneur des Trois-Rivières lance un ordre d'expulsion contre les Jésuites de son Gouvernement, mais le grand vicaire Perrault obtient pour eux un régime de tolérance. Pendant le même temps, les pays catholiques d'Europe appliquent contre les Jésuites un programme de persécution, et ce programme est tout de suite mis en vigueur dans le pays des Illinois: les Jésuites, même ceux qui dépendent du Canada, sont forcés par 
le Gouvernement français de la Louisiane à passer en Europe. Enfin, un jésuite, Roubaud, se retire auprès de Murray et il part en mission politique après avoir obtenu frauduleusement que les Jésuites de Québec en fassent les frais; en Angleterre, Roubaud va travailler contre ses confrères.

Dans cette conjoncture, qu'est-ce que les Jésuites pouvaient espérer des Anglais? Pendant que Murray proposait que les Jésuites soient immédiatement dissous, on présenta des mémoires pour démontrer que ces religieux étaient essentiels à l'éducation de la jeunesse: ce qui était vrai, puisqu'ils dirigeaient l'unique collège classique du pays. Les démarches des Canadiens ne donnèrent aucun résultat: les Jésuites furent condamnés à s'éteindre, et leur collège demeura entrepôt de l'armée. Pouvaient-ils au moins durer assez longtemps pour profiter de circonstances nouvelles qui amèneraient l'Angleterre à modifier sa décision? Là encore, l'espoir est très faible: en cinq ans, les Jésuites ont perdu le tiers de leurs effectifs; au début du Régime du Test, ils ne sont plus qu'en nombre ridicule: 16 prêtres et 5 Frères; le groupe a vieilli: son âge moyen, le plus élevé parmi les Communautés, est de 52.7 ans. Affaiblis en nombre, très âgés comme groupe, tous originaires d'Europe, ayant déjà contre eux les nations officiellement catholiques, les Jésuites ne pouvaient plus durer longtemps dans une colonie protestante. La disparition des Jésuites du Canada est due à un ensemble de circonstances dont les Anglais ne sont pas seuls responsables. Quant à la fermeture définitive de l'unique collège classique de la Nouvelle-France, fermeture qui a précédé de quinze ans la disparition des Jésuites comme groupe, elle est une démolition que l'on peut attribuer à la conquête.

Il va disparaître, et cette fois par le seul verdict du conquérant, une troisième Communauté d'hommes: celle des Récollets. Ces religieux ne possédaient pas de biens personnels; ils ne jouaient aucun rôle qui pût porter envie ou ombrage; à la différence des Jésuites, ils n'étaient pas persécutés dans les pays catholiques. Même si certains de leurs problèmes personnels, portés à la connaissance des autorités militaires, peuvent à cette époque contribuer à compliquer quelque peu la situation de l'Ordre, 
ces religieux forment un groupe propre à s'attirer la sympathie des gouverneurs: à la fin de 1760, les Récollets sont Canadiens dans une proportion de $70.8 \%$, et cette proportion canadienne continue de monter: à la fin de 1764 , elle est de $73.9 \%$ Leurs relations avec les Anglais restent généralement très bonnes et l'on se souvient que leur chapelle, réparée par Murray, est utilisée par les protestants pendant toute la durée du régime militaire et longtemps après. Malheureusement, tous ces bons points ne jouent pas en faveur des Récollets et l'Angleterre décide quand même que ces religieux devront s'éteindre. On s'explique difficilement l'interdit porté contre eux: les archives ne nous apportent aucune lumière là-dessus. Et la Communauté se désagrège ensuite rapidement: à la fin de 1764, sur les 22 Récollets qui restent, il y en a 11, c'est-à-dire la moitié, qui vivent ici et là dans des cures. C'était pour l'Église canadienne la seule façon de combler les vides du clergé séculier, mais c'était en même temps précipiter la décadence de l'Ordre. Les premiers missionnaires du Canada, qui avaient rendu tant de services, se trouvaient condamnés à disparaître, malgré le caractère canadien de leur groupe, sans que l'on puisse expliquer la décision arbitraire du conquérant.

Une quatrième Communauté d'hommes subit péniblement les effets de la conquête, mais cette Communauté va survivre aux événements: le Séminaire de Québec, maison de formation des futurs prêtres, dirigée par quelques séculiers qui mènent la vie commune. La guerre entraîna pour le Séminaire de lourdes pertes: la maison fut littéralement écrasée par les bombes et, pour la reconstruire, il fallut débourser au moins 58,000 livres; la chapelle extérieure dut être restaurée au coût de 5,000 livres; sur les terres du Séminaire, dégâts élevés: maisons du fief Sault-au-Matelot en ruines, la Canardière bouleversée, la côte de Beaupré ravagée d'un bout à l'autre; la seule restauration des fermes et moulins coûtera au moins 23,000 livres. Privé des ressources immédiates de la côte de Beaupré, incapable de faire venir de France l'argent que lui assuraient certaines abbayes, le Séminaire prend beaucoup de temps à se relever: de l'automne de 1759 à l'été de 1761, les prêtres logent ailleurs. Lorsqu'en 1762 , le Bureau de Direction se réunit pour la première fois depuis 
la débandade de 1759 , la maison n'est restaurée que dans une proportion de $5.1 \%$ et le procureur ne trouve en caisse que 180 livres 18 sols 6 deniers d'argent sonnant; les grands séminaristes reviennent de Montréal, où ils avaient continué leurs études, mais le Petit Séminaire demeure toujours fermé. La restauration des fermes et moulins va plus rondement, car il faut accommoder les censitaires et trouver soi-même de quoi se soutenir. Enfin, à l'automne de 1763 , le Séminaire décide de restaurer tout à fait la maison, mais toujours inquiet de l'avenir il précise que si elle ne devait plus servir aux prêtres, le contrat de l'entrepreneur cesserait le jour même de l'avertissement: preuve que le clergé de 1763 craint le pire pour l'Église.

Financièrement, c'est une période difficile que traverse le Séminaire. En comptant les recettes des terres et moulins, les biens de France, les recettes de la maison et autres recettes diverses, nous trouvons pour les années 1759-1764 un revenu brut de 191,560 livres 3 sols 8 deniers, ce qui laisse un surplus de 43,115 livres 18 sols 10 deniers: mais notons bien que dans ce surplus il y a au moins 18,000 livres en argent de papier (or les trois quarts de ce montant ne valent plus rien du tout) et notons surtout qu'il reste encore à rétablir la moitié du Séminaire.

Ce ne furent pas pour le Séminaire les seules graves conséquences de la conquête. Parce qu'elle était dirigée par des Français de France et soumise à l'autorité du Séminaire de Paris, cette institution ne pouvait échapper à quelque exigence soupçonneuse de Murray: le Gouverneur intervient donc personnellement dans la désignation du Supérieur en 1762, il rejette les deux obédiences venues du Séminaire de Paris (et cela à un moment où le Canada n'appartenait pas encore à l'Angleterre), il oblige les directeurs à procéder à une élection; après le traité de Paris, Murray va jusqu'à proposer que les deux Séminaires de Québec et de Montréal soient fusionnés sous l'autorité d'un seul Supérieur qui serait nommé par le Roi. Pendant ce temps, une œuvre importante du Séminaire s'écroulait: la mission des Tamarois, dite de la Sainte-Famille, dans le pays des Illinois; découragé par l'isolement et par la débâcle générale, le grand vicaire Forget du Verger vend les biens de la mission, malgré les protestations 
des habitants, et repasse en France: ce que Mgr Briand qualifiera de «honteuse retraite je dirois même criminelle».

Le Séminaire de Québec va surtout subir une orientation toute nouvelle. Jusqu'à la conquête, il n'acceptait que les candidats à la prêtrise: ces candidats recevaient leur formation au Séminaire et faisaient leurs études au Collège des Jésuites. Lorsque le Petit Séminaire rouvre ses portes à l'automne de 1765 , celles du Collège des Jésuites demeurent fermées: pour assurer la relève, le Séminaire de Québec doit donc s'improviser collège classique et introduire chez lui un enseignement complet qui puisse préparer les jeunes aussi bien aux professions libérales qu'à la prêtrise. Malgré les problèmes difficiles qu'elle pose, cette nouvelle orientation demeure cependant à l'avantage de l'institution québécoise: ce Séminaire, qui n'avait existé sous le régime français qu'en fonction de la préparation du clergé, il est promu collège classique et se voit destiner un rôle de premier plan sur la scène nationale, celui d'assurer la survivance intellectuelle du Canada français.

Des cinq Communautés d'hommes, c'est celle des Sulpiciens qui a subi le moins de dérangement: la guerre ne leur a point causé de dommages matériels et la cession ne change rien à leur mode de vie, sauf en ceci qu'elle les oblige à se séparer des Sulpiciens de Paris pour former désormais une entité distincte: l'œuvre demeure la même. Assurément, les Sulpiciens ont subi quelques effets des événements: l'évêque vient se réfugier chez eux et il faut aussi donner l'hospitalité aux séminaristes québécois; tout cela était de nature à rapprocher deux Communautés qui n'avaient guère l'occasion de se fréquenter. Avec les autorités militaires, les relations des Sulpiciens sont généralement heureuses : ces autorités traitent avec les Sulpiciens comme s'ils étaient reconnus officiellement; un seul orage survient, c'est à l'occasion des mariages mixtes, mais cet orage, de très brève durée, se dissipe par la promotion du Gouverneur. Les Sulpiciens prennent un temps la vedette lorsque leur Supérieur, Montgolfier, est élu évêque de Québec: mais bientôt, sur l'intervention de Murray, Montgolfier remet sa démission comme évêque-élu et comme grand vicaire, et les Sulpiciens se trouvent exclus de l'administration supérieure de l'Église canadienne. 
Leur avenir reste incertain: la capitulation de Montréal leur avait assuré leurs biens, mais ne s'était point prononcée sur la conservation de la Communauté; malgré la proposition, mise de l'avant par Murray, de fusionner les deux Séminaires, les instructions de 1763 ne parlent point des Sulpiciens; leur sort restait en suspens. Un point jouait contre eux: ils étaient tous Français de France; pour se conformer aux nouvelles circonstances, ils se séparent officiellement de leurs confrères de Paris, les seigneurs de Montréal sont désormais les Sulpiciens de Montréal. Notons que cette Communauté d'hommes, qui n'a point souffert de la guerre, a sur les autres Communautés deux avantages qui sont de nature à rendre optimiste: elle est à la fois la plus nombreuse et la plus jeune. A la fin de 1760, elle comptait 30 membres, âgés en moyenne de 43.8 ans, alors que l'âge moyen des Jésuites était de 50.4 ans; à la fin de 1764, la Communauté a quelque peu diminué en nombre, mais ses 27 membres forment toujours la Communauté d'hommes la plus nombreuse et la plus jeune: 45.4 ans. Le nombre et la jeunesse rendaient les Sulpiciens indépendants du recrutement canadien: ils pourront attendre jusqu'en 1767 pour s'agréger le premier Canadien; ils n'en agrégeront un deuxième qu'en 1773: c'est dire jusqu'à quel point l'avenir n'inquiétait pas les Messieurs de Saint-Sulpice.

\section{Problèmes anciens et problèmes nouveaux}

Éprouvée matériellement dans ses temples et dans ses presbytères, menacée de voir se désagréger près de la moitié de ses Communautés de femmes qui sont aux prises avec de très graves difficultés financières, assurée de perdre son Chapitre et de voir s'éteindre les Jésuites et les Récollets, l'Église canadienne doit, de plus, affronter de difficiles problèmes tant sur le plan de ses effectifs que sur le plan politique.

Telle qu'elle se présente à la fin de 1760 , c'est une Église déjà affaiblie numériquement: au début de 1759, elle comptait 196 prêtres (dont un évêque, 9 chanoines, 30 récollets, 31 jésuites, 34 sulpiciens); deux ans plus tard, cette Église, qui n'a plus d'évêque, compte seulement 163 prêtres. Ce clergé appauvri a ses problèmes personnels qui viennent compliquer la situation. 
Dans le Gouvernement de Québec, le grand vicaire Briand trouve beaucoup à se plaindre: «Il n'est presque aucun prêtre que je n'aye Ete obligé de reprendre, si vous En Exceptez 8 a Dix ». Le chanoine Gaillard accorde des dispenses sans posséder les privilèges requis, il intrigue pour se faire nommer grand vicaire: le grand vicaire Briand y va d'une semonce, le Chapitre intervient pour blâmer Gaillard et ce dernier, prétextant ses infirmités, ne se montre jamais plus aux réunions des chanoines. $\mathrm{Ou}$ bien c'est le vieux curé Ménage que Briand doit semoncer parce que Ménage passe outre à certaines formalités; et Briand se heurte encore au Supérieur du Séminaire et au curé de NotreDame-de-Québec. Pour leur indépendance trop prononcée, pour leur trop vif amour de l'aisance ou des biens de la terre, d'autres prêtres s'attirent des réprimandes de Briand: préférant moins de prêtres s'il le faut, mais des prêtres de valeur. Briand menace chaque fois de laisser tomber tout à fait ceux qui font mine de résister. Quelques prêtres donnent dans la chicane, comme ce Duchouquet qui veut sommer un confrère par-devant le juge anglais, à propos d'un fer à hosties; et Briand a fort à faire pour modérer certaines manies procédurières, dont le cas le plus célèbre est celui de Maisonbasse.

Les cas de déchéance extrême sont très rares dans le clergé. Nous avons rencontré ce Raizenne à qui l'on est obligé de retirer sa cure, ce Pierre-Clément Parent qui fait scandale à Saint-Vallier et qui n'en sort que manu militari sur l'intervention de Murray; il y a ce jésuite Roubaud qui, réfugié dans la société protestante, feint de vouloir rentrer en Europe pour s'y convertir et se fait ainsi payer voyage et entretien par les Jésuites de Québec, alors qu'il s'embarque tout simplement dans une mission politique. Ces taches ne doivent pas toutefois nous faire oublier l'admirable dévouement de certains prêtres dans des conditions vraiment héroïques. Si ce clergé avait été, en général, de qualité inférieure, on aurait pu s'attendre à bien des défections; or, Roubaud mis à part (bien qu'il n'ait pas apostasié formellement), l'Église canadienne ne connaît pas une seule apostasie sous le régime militaire: c'est pourtant l'époque où le vainqueur protestant ne demande pas mieux que de voir éclater les faiblesses du 
clergé «papiste», et où l'Angleterre assure officiellement sa protection aux prêtres qui voudront se marier.

Un problème tout à fait nouveau pour l'Église canadienne vient s'ajouter aux problèmes habituels: celui des mariages mixtes contractés par-devant un ministre protestant. Il surgit dès l'automne de 1759. Les autorités religieuses ont été fort embarrassées: le Rituel n'avait rien prévu; l'évêque ne sait trop que faire en 1759 , il ne se prononce ni pour la nullité ni pour la validité du mariage ainsi contracté, il recommande simplement de refuser l'absolution pour punir la Canadienne d'avoir communiqué in divinis avec les protestants; même embarras chez le grand vicaire Briand en 1762; enfin, la Sorbonne se prononce en 1763 pour la validité, mais ces mariages restent illicites et les Canadiennes qui s'en rendent coupables sont soumises à des pénitences secrètes. Ce problème faillit compromettre la cause de l'Église dans le Gouvernement de Montréal: en 1763, après le départ de Montgolfier, le gouverneur Gage somme les Sulpiciens de bénir eux-mêmes ces mariages mixtes; sur leur refus, Gage se fâche, il menace d'expulsion les Sulpiciens; la promotion de Gage à un commandement supérieur met fin à une tempête qui menaçait de prendre des proportions tragiques. Au cours de ces cinq années du régime militaire, nous avons noté d'une façon certaine 14 mariages mixtes: plusieurs textes nous permettent de croire qu'il y en eut beaucoup plus. En tout cas, en plus de contribuer à briser l'homogénéité de cette société catholique, le problème des mariages mixtes était une source d'embarras pour l'Église canadienne.

Ce problème des mariages mixtes se rattache à un problème beaucoup plus vaste: l'établissement du protestantisme dans une colonie qui jusque-là n'a connu, de façon exclusive, que le catholicisme. Sous le régime français, l'Église d'État, c'est l'Église catholique; on tolère les protestants, mais à titre individuel seulement: ils n'ont ni ministre, ni chapelle, ni cimetière, ils ne peuvent exercer aucune fonction publique. La conquête anglaise va non seulement augmenter le nombre des protestants au Canada, mais assurer aux huguenots ce qu'ils n'avaient jamais obtenu en Nouvelle-France: des chapelles, l'inhumation en terre bénite, 
des ministres dont, pendant un certain temps, un ministre de langue française, l'ex-récollet Houdin. Dès l'arrivée des Anglais, les huguenots prennent le haut du pavé: ce sont eux qui, en bien des cas, servent d'intermédiaires entre l'autorité protestante et la population française; c'est même l'un de ces huguenots, François Mounier, qui sera appelé à représenter dans le Conseil de Murray toute la population française du pays: la revanche des huguenots se faisait éclatante. Le renversement des positions est total: alors que seuls les catholiques pouvaient, sous le régime français, accéder aux charges publiques, désormais seuls les protestants pourront, en principe, y avoir accès; l'Église catholique n'est plus qu'à peine tolérée; la religion d'État, c'est le protestantisme.

Dès l'installation des vainqueurs protestants, la position de l'Église canadienne devient fort délicate. Déjà, au cours des opérations militaires, quelques membres du clergé avaient contribué à produire une atmosphère de tension. Certes, nous n'avons trouvé que 14 prêtres, soit une proportion de $7.2 \%$, qui se soient plus ou moins engagés dans le conflit. Quelques-uns jouent un rôle plutôt anodin, mais d'autres se compromettent gravement: Joseph-Basile Parent, curé de l'Ange-Gardien, est forcé d'abandonner sa cure et de se réfugier dans le Gouvernement des Trois-Rivières; Youville-Dufrost, curé de la PointeLévy, reste plusieurs semaines prisonnier à bord d'un navire de guerre; les curés de Charlesbourg et de Beauport, ainsi que des jésuites non identifiés, auraient connu eux aussi l'emprisonnement; deux membres du clergé paient de leur vie leur intervention militaire: l'ecclésiastique Couillard est tué dans une escarmouche; René Portneuf, curé de Saint-Joachim, se fait massacrer à la tête de ses paroissiens dont il dirigeait la résistance. Ce sont là, cependant, des cas isolés; la position de l'Église ne paraît pas en avoir souffert, justement parce que la très grande majorité du clergé se tient en dehors du conflit.

La situation pouvait devenir bien plus difficile lorsque l'Église canadienne aurait à affronter l'autorité militaire ellemême: pour l'Église du Gouvernement de Québec, la première rencontre se produit en septembre 1759, et elle est fort satisfai- 
sante. La capitulation de la ville garantit le libre exercice de la religion et l'évêque peut continuer d'exercer ses fonctions; malade, Mgr de Pontbriand se retire à Montréal, qui est encore sous la domination française, mais l'Église de Québec en territoire occupé ne demeure pas isolée: l'évêque donne toujours les directives nécessaires. Il se comporte d'ailleurs avec la plus grande prudence: il exige que les curés s'en tiennent à leur seul rôle spirituel; il permet aux protestants d'utiliser la chapelle des Ursulines, sans même enlever le Saint-Sacrement s'il faut éviter de froisser les Anglais; il leur laisse enterrer leurs morts dans un cimetière catholique; il défend que l'on parle de religion aux malades protestants et même que l'on administre les derniers sacrements aux catholiques de l'armée anglaise; quand Murray impose aux curés un rôle qui relève plutôt des capitaines de milice, il n'entre pas en discussion avec le Gouverneur et il retire de la Pointe-Lévy le curé qui s'est compromis aux yeux de Murray; quand l'évêque distribue un mandement sur des prières au bénéfice des morts français, il évite de l'envoyer dans la région occupée par les Anglais; lorsqu'il publie un mandement sur certaines dispenses du carême, il demande au grand vicaire Briand de le montrer d'abord au gouverneur Murray. Minutieuse prudence qui s'imposait: le pays était alors transformé en champ de bataille.

La première occupation militaire, 1759-1760, apporte à l'Église de Québec un certain nombre de corvées qui restent légitimes à cause des circonstances et à condition de prendre fin avec les opérations militaires; quelques-unes de ces corvées sont même à l'avantage des institutions qui y ont été soumises: l'Hôtel-Dieu, les Ursulines et l'Hôpital Général en retirent une rémunération profitable et surtout des vivres. Sous cette première occupation, l'Église de Québec conserve une certaine autonomie vis-à-vis le gouverneur Murray : elle est toujours sous la direction immédiate de l'évêque, point capital garanti par la capitulation, et cela est de nature à modérer les empiètements du gouverneur. Malheureusement, il survient un accident qui va causer des complications infinies: Mgr de Pontbriand meurt le 8 juin 1760, donc trois mois avant qu'une capitulation générale vienne étendre 
à tout le pays les privilèges restreints que la capitulation de Québec avait accordés à l'évêque. Murray ne perd pas de temps: huit jours après la mort de Mgr de Pontbriand, il convoque le grand vicaire Briand «pour l'administration des Paroisses qui peuvent être délaissées »; comme il n'y avait que deux paroisses dont la cure se trouvait déserte, il devint évident que le gouverneur de Québec, pourtant occupé à préparer la dernière campagne militaire, se hâte de mettre la main sur l'Église.

La capitulation générale de 1760 consomme le démembrement de la haute administration de l'Église canadienne. Cette capitulation reconnaît les grands vicaires comme tels, mais comme la vallée du Saint-Laurent reste divisée en trois pays bien distincts (chacun dirigé par un Gouverneur indépendant de ses collègues), l'un ou l'autre des grands vicaires n'a juridiction que chez lui et aucun d'eux n'exerce de fait juridiction sur ses confrères, puisque le Chapitre n'a point nommé de vicaire capitulaire; et le Chapitre en eût-il nommé un que ce vicaire capitulaire n'eût joui d'aucune reconnaissance officielle, le traité de Montréal étant muet sur son cas. L'autorité ecclésiastique demeure donc rigoureusement fractionnée: chacune des trois Eglises (québécoise, trifluvienne et montréalaise) existe d'une façon isolée devant l'autorité militaire régionale; chacune est menacée plus que jamais de subir la servitude personnelle d'un gouverneur militaire, celle de Murray, gouverneur de Québec, celle de Burton aux Trois-Rivières ou celle da Gage à Montréal.

Aux Trois-Rivières et à Montréal, cette servitude ne s'impose que d'une façon très occasionnelle et sans affecter sérieusement l'administration ecclésiastique. Le gouverneur de Montréal n'intervient pas dans les cures de sa région. Assurément, il lui arrive de poser ses exigences: il amène Montgolfier à supprimer d'un mandement une déclaration de foi qui serait de nature à 《faire une impression trop sensible sur les esprits »; il se fâche lorsque le Commissaire des Récollets change le supérieur de Montréal, mais il finit par accepter volontiers celui qui a été désigné; il convoque les Sulpiciens pour les sommer de bénir les mariages mixtes et, comme ils refusent, il menace de les expulser du Canada, mais sa promotion vient dénouer heureu- 
sement la tragédie. Aux Trois-Rivières, Burton veut faire lire en chaire un document qui contient une phrase injurieuse à l'adresse des catholiques: le grand vicaire Perrault répond avec fermeté, Burton émonde le texte et se contente de l'afficher à la porte des églises; ce même Burton ordonne aux Jésuites de quitter le Gouvernement des Trois-Rivières, mais Perrault obtient finalement un délai indéfini.

\section{Murray fait marcher l'Église de Québec}

Ce sont là à Montréal et aux Trois-Rivières de rares cas d'ingérence dans l'Église. Il en va bien autrement dans le Gouvernement de Québec où Murray entend bien obtenir et garder le contrôle de l'administration religieuse. Murray avait constaté la puissance que l'évêque exerçait sur son clergé; il veut donc que cette puissance devienne le privilège du gouverneur afin, comme il l'écrit, de tenir les prêtres dans un état de sujétion nécessaire. La capitulation de Québec n'avait pas fait mention des cures; aussi, dès la disparition de l'évêque, Murray convoque-t-il chez lui le grand vicaire Briand et nous constatons que, par la suite, le gouverneur Murray agit tout comme s'il avait pris en mains la direction administrative de l'Église québécoise: à l'automne de 1761, il fait passer Petit de la cure de Deschaillons à celle de Saint-Michel-de-Bellechasse; à la demande de Murray, en janvier 1762, le curé de Berthier-en-bas devient curé de Saint-Vallier; Gatien ne prend possession de la cure de Berthier-en-bas qu'après avoir obtenu l'adhésion de Murray; ce même gouverneur, en août 1762, menace le curé de Saint-Thomasde-Montmagny de l'envoyer à la cure de Grondines; enfin, c'est sur une plainte de Murray que le curé de Saint-Pierre-de-Montmagny est transféré en 1764 à la cure de Lachenaie. Cette prépondérance de Murray est si bien connue que des prêtres y ont recours pour obtenir un ministère de leur choix: le récollet Veyssière réussit de cette façon à demeurer à Saint-Michel-de-Bellechasse malgré les projets du grand vicaire Briand; Pressart, ex-supérieur du Séminaire de Québec, désire faire du ministère à la paroisse Notre-Dame (alors chez les Ursulines) : Briand s'y oppose, mais Pressart va voir Murray et gagne son point. 
Nous retrouvons dans d'autres domaines cette prépondérance de Murray. En 1762, le Séminaire de Paris nomme pour Québec un nouveau supérieur: c'était son droit traditionnel et ce droit demeurait toujours valide puisque le Canada n'appartenait pas encore officiellement à l'Angleterre; mis au courant de cette nomination, Murray convoque les directeurs du Séminaire de Québec et les oblige de procéder à une élection, en excluant d'avance tout prêtre choisi par le Séminaire de Paris. Lorsque Murray a besoin d'une salle chez les Ursulines pour y rendre la justice criminelle, il retient d'abord la salle et ce n'est qu'ensuite qu'il avertit le supérieur ecclésiastique des religieuses. En 1762 , Murray veut que l'on prie pour le Roi d'Angleterre; en cela, rien d'anormal: on priait pour George III aux Trois-Rivières et à Montréal, mais seulement dans les prières du prône. Or Murray veut davantage: avant que le Canada soit cédé à l'Angleterre, Murray veut que le Roi soit nommé dans le Canon de la Messe, tout comme l'était le Roi de France qui, pourtant, détenait ce privilège parce que l'un de ses devoirs officiels était de maintenir le catholicisme. Sans récriminer et bien qu'aux Trois-Rivières et à Montréal on continuât de ne nommer George III qu'au prône, le grand vicaire Briand exécute la volonté de Murray en donnant comme excuse que les ordres sont formels. Chaque fois, Murray trouve devant lui un grand vicaire disposé à obéir.

Et ce grand vicaire ne met jamais en doute les dispositions de Murray. Il ne se scandalise nullement quand Murray somme un curé de comparaître à Québec devant l'autorité militaire, avec défense de conférer avec qui que ce soit auparavant. Briand fait même plus pour encourager l'ingérence de Murray: comme le vicaire Parent refuse de sortir de Saint-Vallier, Briand invite l'autorité militaire et protestante à servir de bras séculier. Il a en Murray une telle confiance qu'il propose même que l'élection de l'évêque se fasse en présence du gouverneur et que, si ce dernier n'est pas satisfait, on recommence l'élection.

Le gouverneur Murray (et c'est peut-être ce qui explique le comportement de Briand) ne souhaitait pas la disparition de l'Église canadienne; il voyait tout simplement en elle un puissant organisme qu'il serait fort utile de contrôler sévèrement: en lui 
donnant un caractère plus national et en faisant nommer les curés par le Roi ou par le Gouverneur, Murray comptait la rendre tout à fait docile. Il avait été assez heureux, depuis les débuts du régime militaire, pour tenir bien en mains l'Église de Québec. C'est pourquoi, quand vint le temps de régler le problème religieux de la nouvelle colonie, Murray proposa une solution conforme à sa propre politique: l'Église canadienne serait toujours administrée par des vicaires généraux, les deux Séminaires seraient fusionnés en un seul et le Supérieur serait nommé par le Roi, on renverrait immédiatement les Jésuites avec une pension, la nomination des curés appartiendrait au Gouvernement; bref, cette Église sans évêque, parfaitement soumise aux obédiences politiques, demeurerait un instrument fort commode de domination.

Ce programme accablant est proposé par le gouverneur Murray à une époque où l'Église canadienne subit une diminution désastreuse dans ses effectifs. En janvier 1759, elle comptait 196 prêtres : à la fin de décembre 1764, elle n'en compte plus que 137, ayant donc perdu dans le bref espace de cinq ans, le tiers de ses effectifs. Pour combler ce vide, on ne peut plus compter sur le recrutement de France et l'on ne trouve au pays qu'une dizaine de grands séminaristes qui doivent attendre la venue encore toute problématique d'un évêque. Chez les Communautés d'hommes, la situation devient désastreuse: le Chapitre a terminé son histoire; les Jésuites, déjà réduits au petit nombre de 16, ont reçu l'ordre de s'éteindre; les Récollets, dont la moitié ne mènent plus la vie conventuelle, devront eux aussi s'éteindre; le Séminaire de Québec, qui a subi pour plus de 85,000 livres de dommages, ne doit plus compter que sur lui-même, puisque le Séminaire de Paris n'a plus le droit de lui envoyer du renfort, et il doit, de plus, modifier profondément sa formule en créant chez lui de toute pièce un collège classique qui remplacera celui que les autorités anglaises ont transformé en entrepôt. Des cinq Communautés d'hommes, il n'y a vraiment que celle des Sulpiciens qui s'en tire avec le moins possible d'inconvénients. Partout, la pénurie du clergé est très grave: il n'y a plus que 3 prêtres dans l'immense région des Illinois, 3 prêtres dans tout le pays des Grands Lacs; dans l'ancien Gouvernement des Trois-Rivières, 
la moitié des centres de population sont sans prêtre résidant; un seul prêtre doit desservir toute la rive nord depuis les Éboulements jusqu'aux Sept-Îles; sur la rive sud, depuis les TroisPistoles jusqu'à la Baie-des-Chaleurs, un seul prêtre, et encore est-ce un septuagénaire! dans toute l'Acadie (continentale, péninsulaire et insulaire), il n'y a plus là aussi qu'un seul prêtre.

\section{LE DRAME ÉPISCOPAL}

C'est donc dans cette atmosphère très pessimiste que Murray propose pour l'Église canadienne un modus vivendi qui correspond exactement à un modus moriendi. Heureusement pour l'Église, ce n'est pas tout à fait ce modus vivendi que l'Angleterre va accepter: pour subsister, l'Église canadienne avait essentiellement besoin d'un évêque, et la métropole adoptera là-dessus une politique de laisser-faire. En effet, peu après le traité de Paris, le doyen Lacorne s'était rendu en Angleterre pour y discuter du problème capital, celui de l'épiscopat. L'Angleterre ne pouvait plus permettre au Roi de France de nommer l'évêque de Québec; elle ne pouvait non plus laisser Rome s'ingérer dans les affaires du Canada; et les lois de la Grande-Bretagne ne permettaient pas au Roi de nommer un évêque catholique. Il ne restait qu'une solution: faire élire un évêque par le Chapitre; pressée par les ambassades catholiques, l'Angleterre accepta officieusement cette solution. Et le 15 septembre 1763, les chanoines du Canada désignent d'une voix unanime le sulpicien Montgolfier. Choix excellent, pensent-ils : le Séminaire de Montréal est la seule institution capable de soutenir convenablement un évêque; de plus, c'est Gage, gouverneur de Montréal et ami de Montgolfier, qui a le plus de chance de devenir gouverneur général du pays; politiquement et matériellement, l'épiscopat se trouvait assuré. Pour éviter toute ingérence malencontreuse, l'élection épiscopale se fait dans le plus grand secret et tout de suite Montgolfier s'embarque pour l'Europe.

Il surgit bientôt des obstacles. Même si Rome refuse de reconnaître au Chapitre le droit d'élire l'évêque, ce n'est pas du Saint-Siège que vient l'opposition bouleversante, car Montgolfier est accepté à Rome à condition que sa nomination vienne 
du Saint-Siège et non du Chapitre: le grand obstacle, celui qui change tout, vient du pouvoir politique. Contrairement aux prévisions, c'est Murray qui recevait la charge de gouverneur général: or, par deux fois, il avait présenté un dossier contre Montgolfier au cas où celui-ci figurerait sur la liste des candidats à la mître. Tout en reconnaissant que les accusations de Murray étaient sans fondement, le Secrétaire d'État Halifax ne voulut point imposer au gouverneur général un évêque qui ne fût point de son goût. Revenu au Canada, Montgolfier démissionne donc comme évêque-élu en recommandant Briand; il démissionne même comme grand vicaire et Murray se vante de lui avoir nommé un remplaçant. Le 11 septembre 1764, les chanoines recommencent l'élection épiscopale: le vote désigne Briand. Au nouvel élu, le Chapitre remet un acte d'élection et un acte de présentation, laissant à Briand le soin d'utiliser l'un ou l'autre afin de ne pas prolonger la discussion avec le Saint-Siège.

Briand était le candidat personnel de Murray: le gouverneur l'avait maintes fois recommandé, il l'avait fort louangé au détriment de Lacorne et de Montgolfier, il en avait éprouvé la constante soumission. On pouvait donc au moins espérer que la protection de Murray hâterait le règlement du problème épiscopal. Cependant, depuis qu'au printemps de 1764 le Secrétaire d'État Halifax avait sacrifié Montgolfier, le prestige de Murray baissait rapidement en Angleterre, et Briand, qui croyait tout réussir à cause de Murray, ne rencontre plus que des difficultés: arrivé à Londres en novembre 1764, ce n'est qu'à la fin de $\mathbf{1 7 6 5}$ que Briand obtient de l'Angleterre la confirmation semi-officielle du laisser-faire, et encore le mérite de ce succès revient-il surtout aux adversaires de Murray. Quand l'évêque Briand remonte le Saint-Laurent en juin 1766, il rencontre un navire qui ramène Murray en Angleterre; celui que Murray avait imposé à l'épiscopat entre en charge au moment précis où disparaît le protecteur autoritaire et encombrant. Le gouverneur Murray, par son ingérence constante dans les affaires de l'Église, avait quand même créé un précédent, et l'on sait la force du précédent dans les mœurs britanniques: pendant trois quarts de siècle, on verra 
les gouverneurs intervenir pour contrôler non seulement les nominations épiscopales, mais encore les nominations aux cures.

Ce régime de servitude, imposé à l'Église canadienne par un gouverneur, est un fait entièrement nouveau dans notre histoire. Certes, sous le régime français, l'Église subissait la servitude du Roi de France: officiellement chargé par le Pape de veiller au soutien du catholicisme, le Roi de France nommait l'évêque; il nommait aussi le doyen et le grand-chantre du Chapitre; il contrôlait l'autorité religieuse pour l'empêcher d'empiéter sur le profane; dans une colonie où presque toutes les institutions religieuses étaient maintenues dans l'existence par les subsides de l'État, le Roi de France déterminait lui-même le status et la population maxima des Communautés; mais tout cela se rattachait à la série des devoirs officiels du Roi Très Chrétien pour le soutien de la cause de l'Église. Et il faut reconnaître que Louis XIV et Louis XV (quelle que fût leur vie privée) ont toujours vu de près à ce que cette cause soit magnifiquement servie au Canada. Mais ce qu'il importe surtout de remarquer ici, c'est que, sous le régime français, le Roi de France n'a jamais délégué aucun gouverneur pour accomplir les devoirs du Trône vis-à-vis de l'Église: l'Église canadienne a conservé toute liberté en face des gouverneurs français, et lorsque l'un ou l'autre a voulu s'ingérer dans les affaires religieuses (même quand c'était à la demande de l'évêque, comme cela s'est produit dans un changement de curés), le Roi de France est aussitôt intervenu pour rabrouer ce gouverneur qui osait dépasser le domaine profane. Le contrôle de l'Église était affaire non pas d'un gouverneur, individu dont les intérêts personnels pouvaient causer préjudice, mais affaire de la Couronne. Or, en plus de passer tout à coup sous la servitude d'un Roi protestant qui doit en principe lutter contre le catholicisme, l'Église canadienne entre sous la servitude personnelle d'un gouverneur protestant qui entend bien soumettre l'Église à ses caprices personnels: le rejet de Montgolfier et les complications qui s'ensuivent illustrent bien cet état de choses. 


\section{A L'ORIGINE DE LA PUISSANCE DU CLERGÉ SÉCULIER}

Il faut bien reconnaître cependant que ce régime de servitude n'a pas été seulement une source de graves dangers ou d'inconvénients: l'Église canadienne en a retiré à longue échéance d'importants avantages. En acceptant avec soumission le comportement de Murray dans les affaires religieuses, le grand vicaire Briand n'avait pour ainsi dire obtenu en retour aucune faveur immédiatement tangible pour la cause religieuse: il n'empêche que le programme mis en œuvre par Murray, confirmé en partie par l'Angleterre et poursuivi selon les circonstances, a servi sous certains aspects à fortifier la position de l'Église canadienne.

En effet, à cause de ce programme, le clergé acquerra bientôt une complète homogénéité. Homogénéité d'abord du point de vue de l'origine: ce mélange de prêtres français et de prêtres canadiens n'avait pas toujours été sans quelques disputes et, pour régler ce problème, Mgr Dosquet avait proposé d'entourer, pour ainsi dire, de prêtres français les prêtres canadiens qu'il trouvait trop indépendants; à la fin du régime français, les uns et les autres sont en nombre égal, mais l'épiscopat appartient à un Français de France, deux des trois grands vicaires du SaintLaurent sont Français, les supérieurs de Communautés d'hommes sont Français. Déjà, sous le Régime militaire, une évolution très marquée se produit: à l'automne de 1764 , l'évêque proposé est un Français de France, mais un Français qui est fixé au Canada depuis 23 ans; les trois grands vicaires sont Canadiens; l'ensemble du clergé n'est encore Canadien que dans une proportion de $55.9 \%$, mais comme les Jésuites et les Récollets sont appelés à s'éteindre et que le clergé séculier est Canadien dans une proportion de $88.1 \%$, l'Église canadienne progresse rapidement vers l'homogénéité d'origine. Murray avait écrit en 1762: "Si l'état ecclésiastique ne se composait que de natifs, ceux-ci deviendraient bientôt dociles et satisfaits »; plus tard, il déclarait que, aussi longtemps qu'il serait gouverneur, il ne laisserait venir au Canada aucun prêtre français. Et l'on sait que, pendant une trentaine d'années, le recrutement français restera nul parce qu'interdit. Privée du recrutement de France, qui aurait tout 
de même pu contribuer à élever le niveau du clergé, l'Église se renouvelle à même ses ressources autochtones; à la fin du siècle, elle est une Église parfaitement canadienne: cette transformation rapide est le fait de la politique religieuse des autorités anglaises.

Cette même politique des autorités anglaises a assuré à l'Église canadienne une autre homogénéité: celle d'une Église composée presque uniquement de prêtres séculiers. ${ }^{1}$ Sous le régime français, la présence au Canada de multiples groupes à l'intérieur de l'Église (Jésuites, Récollets, Sulpiciens et séculiers) avait eu pour effet volontaire, de la part du Roi de France, d'équilibrer les influences religieuses dans la colonie; mais cela avait en même temps pour effet de rendre plus difficile, à qui voudrait le prendre, le contrôle de cette Église. A partir de 1764, ce jeu d'équilibre est détruit systématiquement: les Sulpiciens, éliminés de la haute administration religieuse et tenus longtemps, comme corps constitué, dans l'incertitude de leur avenir, devront pendant plusieurs années se contenter tout juste d'exister; quant aux Jésuites et aux Récollets, jusque-là fort influents, ils reçoivent l'ordre de s'éteindre. Il ne reste plus en somme que le clergé séculier: dans le drame ecclésiastique, c'est lui qui devient le maître incontesté de la scène, c'est lui désormais qui constitue, pour ainsi dire, toute l'Église canadienne. Cette prépondérance absolue que le clergé séculier n'avait jamais eue sous le régime français, qu'il acquiert d'un seul coup et qu'il gardera très facilement pendant plus d'un siècle, il la doit à la Conquête.

Et non seulement le clergé séculier peut désormais être en somme, à lui seul, toute l'Église canadienne, mais il devient pour longtemps le seul organisme intermédiaire entre l'État et le peuple. Dépendants de la Couronne par l'entremise de l'Évêque mais indépendants du Gouverneur, les prêtres du régime français avaient dû, sauf en de très rares cas, se limiter rigoureusement à leur rôle religieux auprès des fidèles; dans la société,

1 Rappelons ici que, dans ce tableau de 1764 , nous ne comptons pas parmi les séculiers les 27 Sulpiciens et les 4 Messieurs du Séminaire de Québec, parce qu'ils vivent en Communauté. 
les prêtres constituaient un groupe, mais ce groupe n'était pas au-dessus d'un autre au nom de qui il pouvait parler à l'autorité civile ni qu'il pouvait diriger à d'autres fins que des fins religieuses. Après la Conquête, il n'existe qu'une classe organisée, qu'un seul organisme vraiment représentatif des Canadiens français, qu'un seul groupe qui puisse exercer une influence décisive sur le peuple: c'est le clergé séculier. En précipitant le caractère canadien de ce clergé séculier et en lui laissant la prépondérance absolue à l'intérieur de l'Église, à condition évidemment de le soumettre au contrôle rigoureux de l'État, les autorités anglaises trouvaient là l'intermédiaire le plus efficace entre elles et le peuple. C'est seulement à partir de la Conquête que les prêtres peuvent, sur le plan social, aller au delà de leur rôle religieux et c'est à cause de cette nouvelle situation qu'ils pourront, par exemple, prendre l'initiative de diriger l'effort du peuple contre l'assimilation anglaise: ce que Murray n'avait certes pas prévu... En tout cas, la servitude imposée à l'Église canadienne avait pour effet, plus ou moins immédiat, de donner à ce clergé séculier une puissance qu'il n'avait jamais connue à l'intérieur de la colonie.

Tout cela, évidemment, pouvait échapper à un observateur de 1764, moins bien placé que nous le sommes aujourd'hui, pour juger des suites de la politique religieuse des autorités anglaises. Des observateurs comme le grand vicaire Perrault et le prêtre Gravé sont forts pessimistes sur l'avenir de l'Église canadienne, et leur pessimisme est bien légitime. Que voient-ils, en effet ? Trois des sept Communautés de femmes touchent de près à la catastrophe; le Chapitre, les Jésuites et les Récollets sont condamnés à disparaître; l'unique collège classique reste fermé et le Séminaire de Québec, jusque là maison de formation religieuse, devra s'improviser collège; dans le bref intervalle de cinq ans, l'Église a perdu le tiers de ses effectifs et elle n'a plus le droit de se recruter en France; auparavant Église d'État dans une colonie officiellement catholique, elle tombe au rang d'Église tout juste tolérée dans une colonie officiellement protestante; asservie jusque là mais en même temps soutenue par un roi catholique, elle passe sous la tutelle d'un simple gouverneur protestant qui 
vient d'en profiter pour imposer à l'épiscopat un candidat de son choix, aux dépens de celui que le Chapitre avait librement élu. Pour un observateur de 1764, cette Église affaiblie dans ses effectifs, ébranlée dans ses institutions et dominée par les autorités protestantes entrait dans la période la plus difficile de son histoire.

\author{
Marcel TRUDEL, \\ Professeur titulaire d'histoire \\ du Canada à l'Université \\ Laval (Québec)
}

N.B. Prière de se rappeler qu'avec la présente livraison de juin, l'abonnement à la Revue, pour l'année 1957-1958, devient échu.

N.B. Prendre note également que le no de mars de 1957, qui contiendra l'index général des dix ans de la Revue - no en retard bien malgré nous - paraîtra d'ici quelques semaines. 